\title{
Ovarian metastasis from non-small cell lung cancer with ALK and EGFR mutations: A report of two cases
}

\author{
ROSEMARY T. MUSHI ${ }^{1 *}, Y_{U M E I}$ YANG $^{2 *}$, QIAN CAI $^{1}$, RUIGUANG ZHANG $^{1}$, \\ GANG WU ${ }^{1}$ and XIAORONG DONG ${ }^{1}$ \\ ${ }^{1}$ Cancer Center, Union Hospital, Tongji Medical College, Huazhong University of Science and Technology; \\ ${ }^{2}$ Department of Respiratory Medicine, Tianyou Hospital, Wuhan University of Science and Technology, \\ Wuhan, Hubei 430000, P.R. China
}

Received April 26, 2015; Accepted August 12, 2016

DOI: $10.3892 / 01.2016 .5292$

\begin{abstract}
Ovarian metastasis from non-small cell lung cancer (NSCLC) is a rare condition. The current study presents the cases of 2 female patients aged 38 and 47 years old, respectively, who were initially diagnosed with NSCLC adenocarcinoma on histology. Both patients initially presented with chest pain and a cough, and subsequently developed ovarian metastases following multiple treatments. The 38-year old patient exhibited an epidermal growth factor receptor mutation, confirmed by scorpion/amplified refractory mutation system analysis from a lung biopsy. The 47-year old exhibited an anaplastic lymphoma receptor tyrosine kinase (ALK) rearrangement, revealed by fluorescence in situ hybridization analysis of the breast tissue biopsy, confirming a diagnosis of ALK rearrangement-positive NSCLC. These patients developed ovarian metastasis in the course of the disease. The current study reports the diagnostic challenges and clinical management of the disease, and provides a review of the literature.
\end{abstract}

\section{Introduction}

Bronchogenic carcinoma is the leading cause of tumor-related mortality in developed countries (1). Lung cancer was associated with $\sim 1,590,000$ deaths in 2012, and it is currently the leading cause of cancer-associated mortality worldwide. Non-small cell lung cancer (NSCLC) accounts for $\sim 85 \%$ of all lung cancer cases (2). Women with metastatic ovarian

Correspondence to: Professor Xiaorong Dong, Cancer Center, Union Hospital, Tongji Medical College, Huazhong University of Science and Technology, 56 Wujiadun Street, Wuhan, Hubei 430000, P.R. China

E-mail: hustwhuh@126.com

*Contributed equally

Key words: non-small cell lung cancer, ovarian metastasis, epidermal growth factor receptor mutation, anaplastic lymphoma receptor tyrosine kinase rearrangement-positive adenocarcinoma from the lung have been found to have a mean age of 46 years (3), with disease onset at a young age also being a prominent characteristic of anaplastic lymphoma receptor tyrosine kinase (ALK) rearrangement-positive NSCLC (4). The ovaries are an uncommon location for metastasis from lung cancer, and few such cases have been reported. The 2 patients in the present study developed ovarian metastasis at different times in their illnesses, and 1 patient developed breast metastasis. Ovarian metastasis from lung cancer represents only $2-4 \%$ of all ovarian metastatic masses. This frequency, however, is increasing due to the rising incidence of lung cancer in women (3). Secondary metastatic ovarian tumor occurrence is variable and depends on a number of factors, including the accuracy of the pathological diagnosis, the completeness of the staging and possible genetic patterns. In total, 7 cases of ovarian metastasis of lung cancer have been reported (5). In order to find a suitable treatment strategy, an indicator for ovarian metastasis from lung cancer is essential (5). Although, there are diverse clinical indicators for cancer prognostic evaluation, patients who share the same clinical features can have quite different clinical outcomes. Nowadays, with the development of gene profiling techniques, such as microRNA microarray and reverse transcription-quantitative polymerase chain reaction, novel biomarkers are intended to be used as prognostic factors combined with traditionally clinical features (6-8).

The current study presents two female NSCLC patients who developed ovarian metastases during their clinical courses. The patients' epidermal growth factor receptor (EGFR) or ALK mutation status was analyzed after the diagnoses of ovarian metastases, and these two patients received different therapies. This report also provides a brief review of the literature regarding ovarian metastases of lung cancers, providing some clues into the clinical management of this disease. Written informed consent was obtained from the patient or the patient's family for the publication of this study.

\section{Case report}

Case 1. A 38-year-old female was admitted to the Cancer Center of Union Hospital (Tongji Medical College, Huazhong University of Science and Technology, Wuhan, China) in May 2011 due to lower back pain that had persisted for 

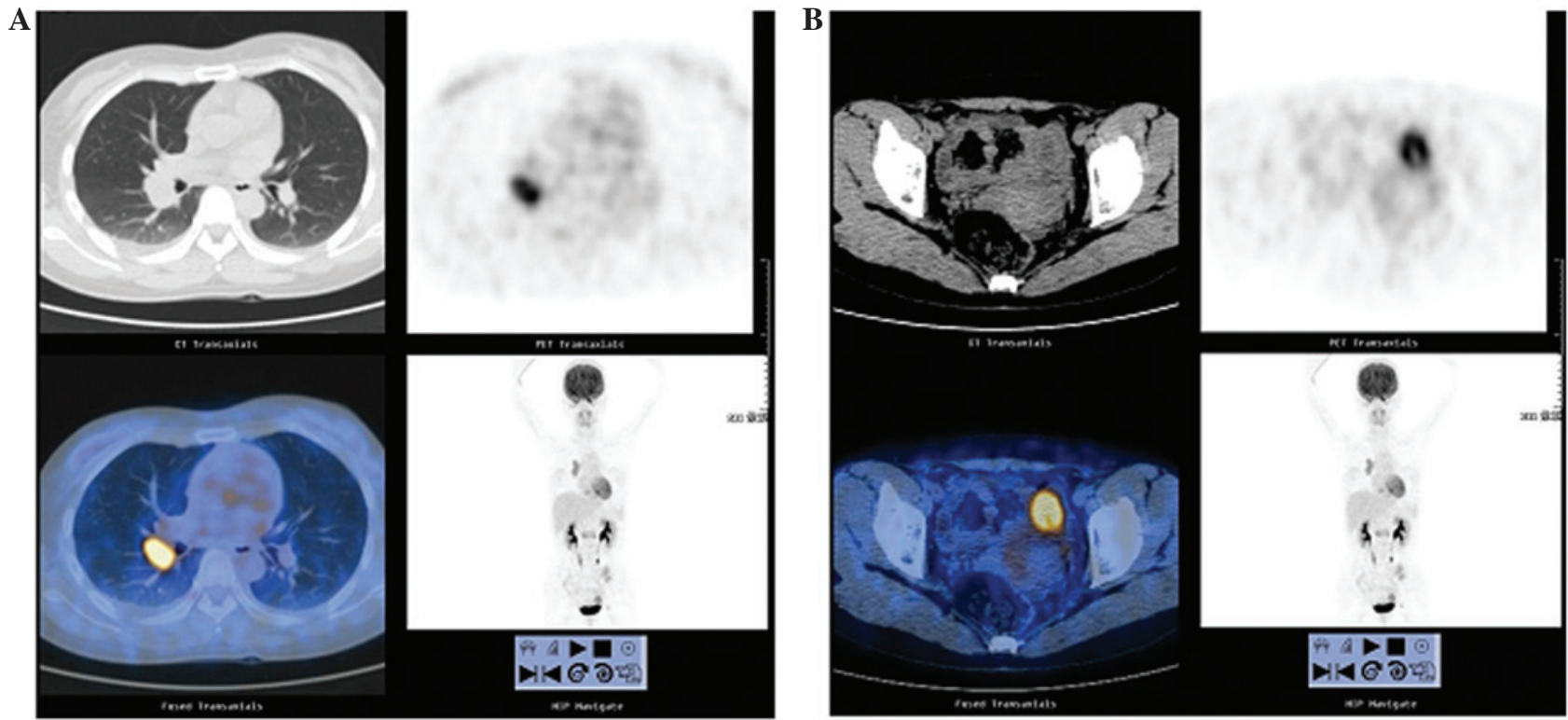

Figure 1. PET-CT diagnosis of ovarian metastasis of lung cancer. (A) PET-CT of the whole body revealing a high metabolic signal at the right upper lobe of the lung. (B) PET-CT of the whole body revealing a high metabolic signal at the left ovary. PET-CT, positron emission tomography-computed tomography.
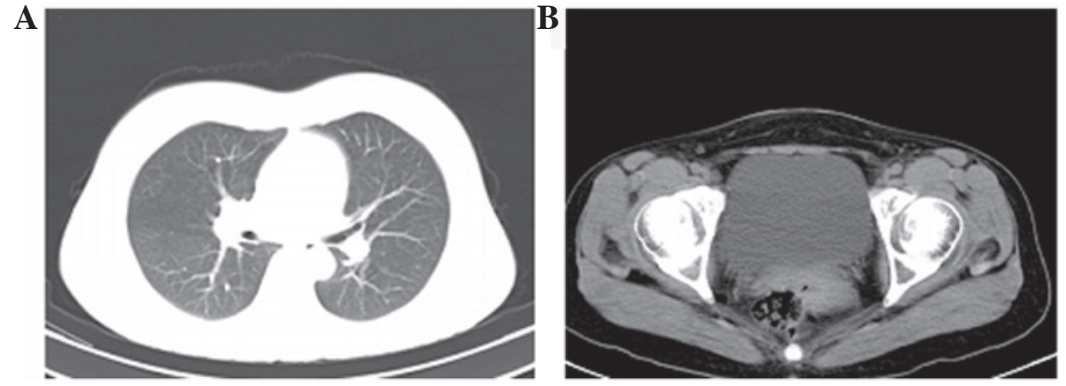

Figure 2. The response of the primary disease and ovarian metastasis on CT scan following Tarceva treatment. (A) CT scan showing disappearance of the primary right lung mass. (B) CT scan showing complete remission of the left ovarian mass. CT, computed tomography.

$\sim 4$ months. At 1 month prior to admission, the patient reported worsening of this symptom, with pain radiating to the left hip and reduced movement of the left leg, which was not associated with numbness or relieved by lying down. There was no history of trauma, fever, abdominal pain, vomiting, headaches, coughing or chest pain. The patient had no history of smoking, and there was no family history of a similar presentation or a significant past medical history.

Positron emission tomography-computed tomography (PET-CT) of the whole body revealed a high metabolic signal at the right upper lobe of the lung (Fig. 1A), at the left ovary (with enlarged right hilar lymph nodes) (Fig. 1B), and in multiple bone areas. The patient also underwent a CT-guided biopsy of the mass on the right upper lobe of the lung, which showed a middle-grade differentiated, glandular structure indicative of primary lung adenocarcinoma. Furthermore, EGFR mutation analysis was performed using scorpion/amplified refractory mutation system technology, revealing 21 exon mutations. The patient was finally staged as T4N1M1 according to the tumor-node-metastasis classification system (9). The patient was started on $150 \mathrm{mg} /$ day Tarceva, with concurrent radiation therapy to lumbar vertebrae 1 and 2 , and to the left iliac bone to a dose equivalent to $40 \mathrm{~Gy}$ in 20 fractions. After $\sim 5$ months, the mass in the right upper lobe of the lung had decreased in size until near complete remission (CR) was achieved (Fig. 2), with normal tumor marker levels. The patient was discharged to continue with Tarceva therapy and was advised to attend regular follow-up clinics. At $\sim 9$ months after discharge, follow-up chest and pelvic CT scans showed a relapse on the right ovary, but stable disease (SD) on the upper right lung (Fig. 3).

The patient was started on $500 \mathrm{mg} / \mathrm{m}^{2}$ pemetrexed plus $75 \mathrm{mg} / \mathrm{m}^{2}$ cisplatin (both administered on day 1 of the cycle), with a partial response (PR) after 6 cycles, followed by radiotherapy to the left ovary consisting of $50 \mathrm{~Gy}$ in 25 fractions. The patient was then discharged to attend follow-up clinics at a local hospital. In February 2013, the patient reported to the local hospital with headaches, nausea, vomiting and fainting episodes. A CT scan was performed, which revealed brain metastasis. The patient was referred back to the Cancer Center and readmitted. Magnetic resonance imaging (MRI) revealed multiple brain metastasis and whole-brain radiation was consequently performed to $36 \mathrm{~Gy}$ in 12 fractions. Following completion of the therapy, the patient returned home, where she succumbed 2 months later. Written informed consent was obtained from the patient for the publication of this study. 

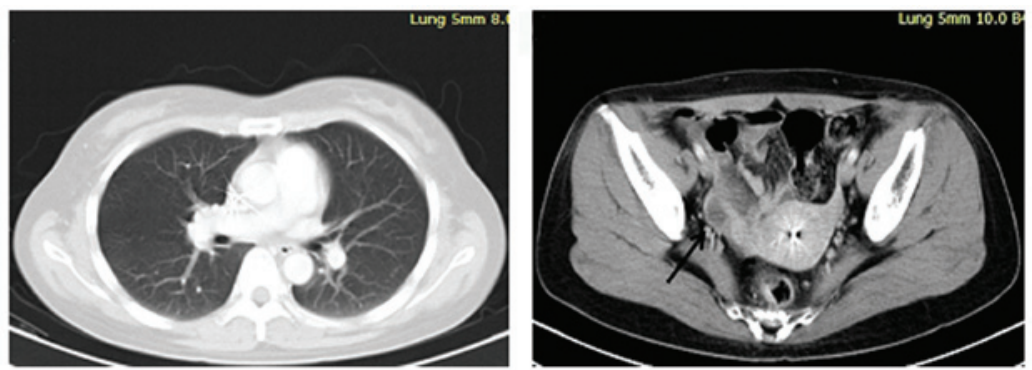

Figure 3. Computed tomography scan of the pelvis showing right ovarian metastasis.

Case 2. In August 2010, a 47-year-old female presented at the Cancer Center of Union Hospital due to a cough that had persisted for 7 months. A chest CT scan revealed a mass on the right lung. PET-CT of the whole body was also performed, which showed high metabolic signals on the right lung and right supraclavicular lymph node (Fig. 4). The cytology of the lymph node revealed metastatic adenocarcinoma and the patient was diagnosed with right lung adenocarcinoma, clinical stage T4N3M0. Chemotherapy with $1,000 \mathrm{mg} / \mathrm{m}^{2}$ gemcitabine (on days 1 and 8) plus $75 \mathrm{mg} / \mathrm{m}^{2}$ cisplatin (on day 1) was initiated. After 2 cycles, the response was SD and therefore, the chemotherapy was changed to $500 \mathrm{mg} / \mathrm{m}^{2}$ pemetrexed and $75 \mathrm{mg} / \mathrm{m}^{2}$ cisplatin (both administered on day 1 of the cycle). After 4 more cycles of chemotherapy, the patient was treated with maintenance therapy of $150 \mathrm{mg} /$ day erlotinib. In April 2012, a follow-up ultrasonic pelvic examination revealed a mass in the right ovary (Fig. 5). A bilateral salpingo-oophorectomy (BSO) was performed, with the biopsy result showing moderately-differentiated adenocarcinoma of the right ovary (Fig. 6A and B) with EGFR wild-type. Immunohistochemistry showed strong reactivity for cytokeratin (CK)7, napsin A (Fig. 6C) and thyroid transcription factor 1 (TTF-1) (Fig. 6D), while the tumor was negative for cancer antigen 125. A diagnosis of metastatic adenocarcinoma of the lung was formed.

Subsequent to the BSO, the patient received 2 cycles of pemetrexed alone, followed by $150 \mathrm{mg} /$ day erlotinib. After 3 months, the patient began experiencing back pain, which worsened within 1 month. Upon visiting a local hospital, bone emission computed tomography was performed showing multiple bone metastases. The patient was readmitted to the Cancer Center 1 month later where radiotherapy was administered to the T6-T8 vertebrae at a dosage of $30 \mathrm{~Gy} / 10$ fractions. This was followed by 3 cycles of $75 \mathrm{mg} / \mathrm{m}^{2}$ docetaxel (on day 1), but the disease progressed to brain metastasis according to MRI of the brain. The patient underwent whole-brain radiotherapy with a total dose of $36 \mathrm{~Gy} / 12$ fractions. After 2 weeks, multiple right breast masses were detected and a biopsy was performed, which showed metastatic adenocarcinoma (Fig. 7A and B). Immunohistochemistry showed strong reactivity for CK7, TTF-1 and $\mathrm{CK}$, while the tumor was negative for estrogen receptor, progesterone receptor, human epidermal growth factor receptor-2, napsin A, cluster of differentiation 56, CK20 and villin. In addition, fluorescence in situ hybridization analysis revealed the presence of ALK rearrangement (Fig. 7C), confirming a diagnosis of ALK rearrangement-positive NSCLC with breast metastasis. Moreover, EGFR analysis revealed the presence of the wild-type gene. The patient achieved a PR once started on

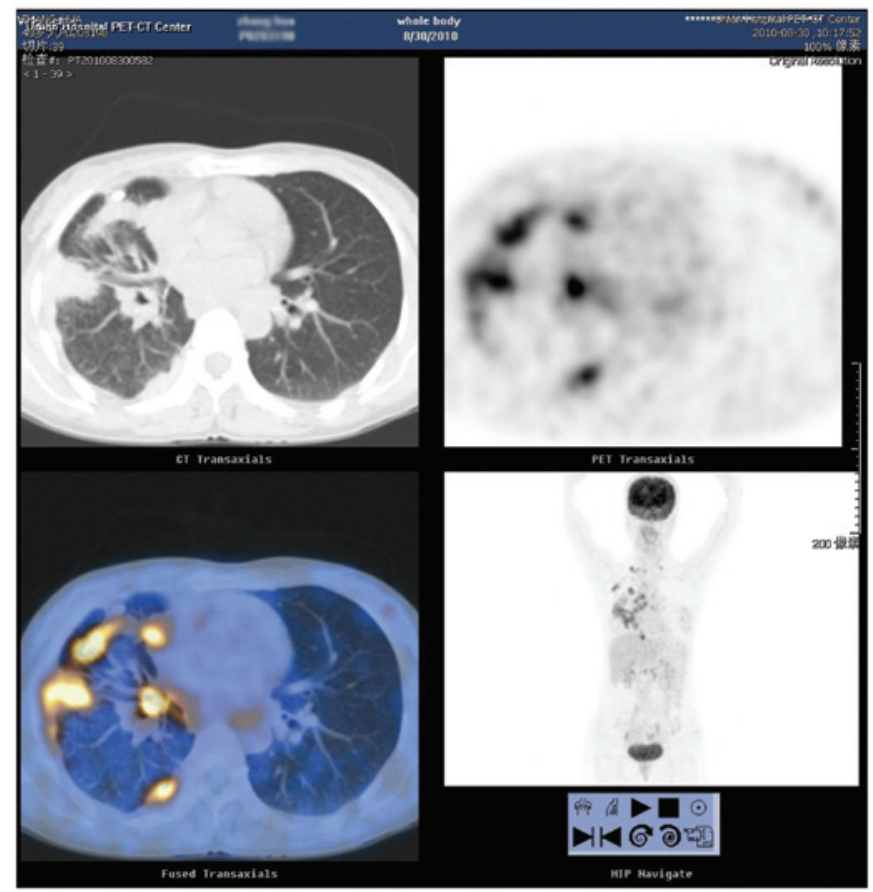

Figure 4. Positron emission tomography-computed tomography of the whole body showing high metabolic signals on the right lung.

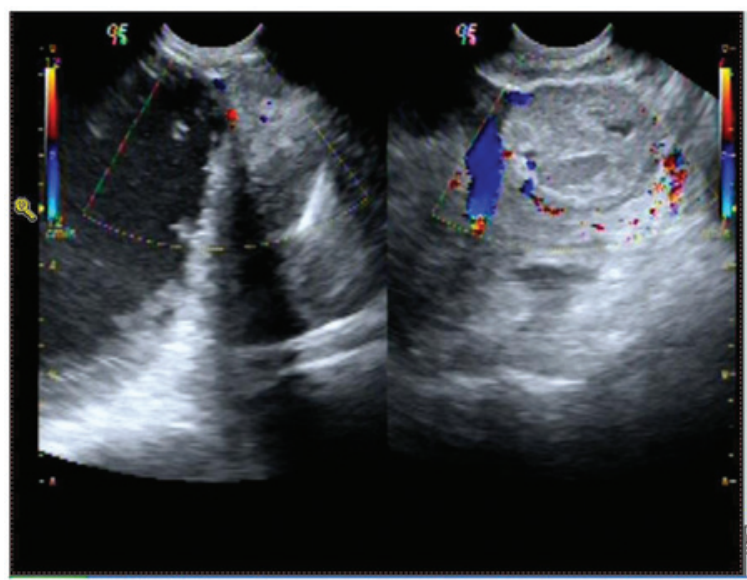

Figure 5. Ultrasonic pelvic examination showing a right ovarian mass.

twice daily $250 \mathrm{mg}$ crizotinib (Fig. 8). The patient has shown no evidence of progression during regular follow-up visits for 1 year since crizotinib treatment. Written informed consent was obtained from the patient for the publication of this study. 

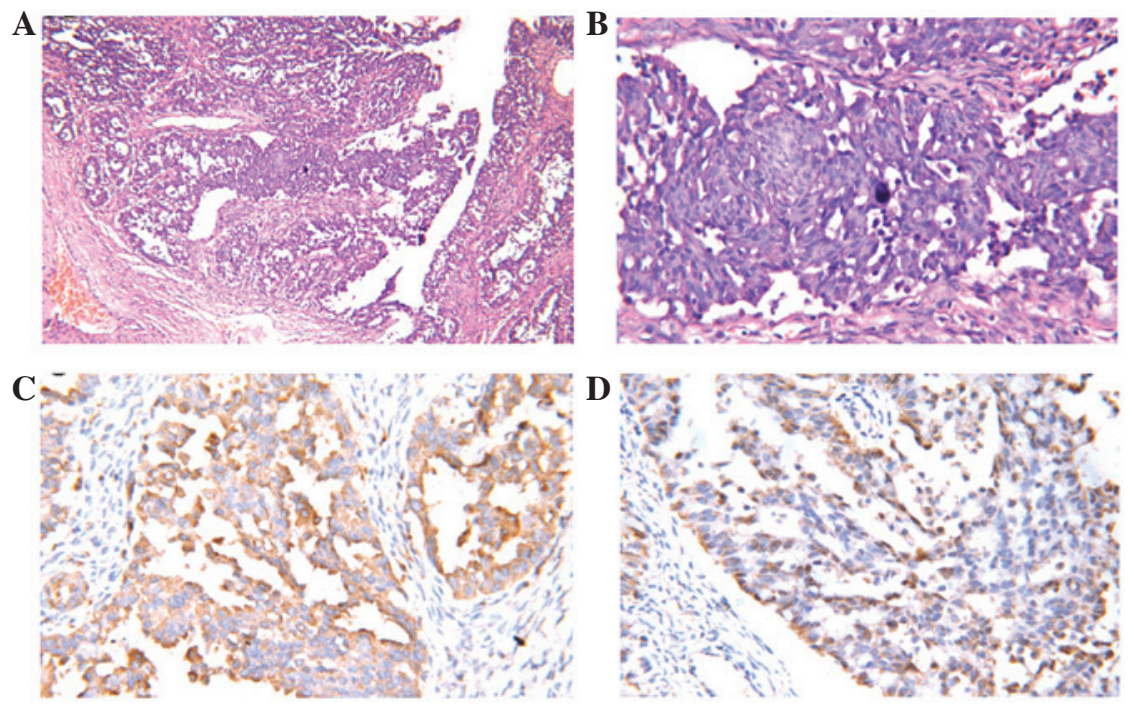

Figure 6. Ovarian specimen obtained from tumor bilateral salpingo-ophorectomy showing moderately-differentiated adenocarcinoma of the right ovary. (A) Hematoxylin and eosin staining; original magnification, x40. (B) Hematoxylin and eosin staining, original magnification, x400. Immunohistochemical analysis showing strong reactivity for (C) napsin A and (D) thyroid transcription factor 1.
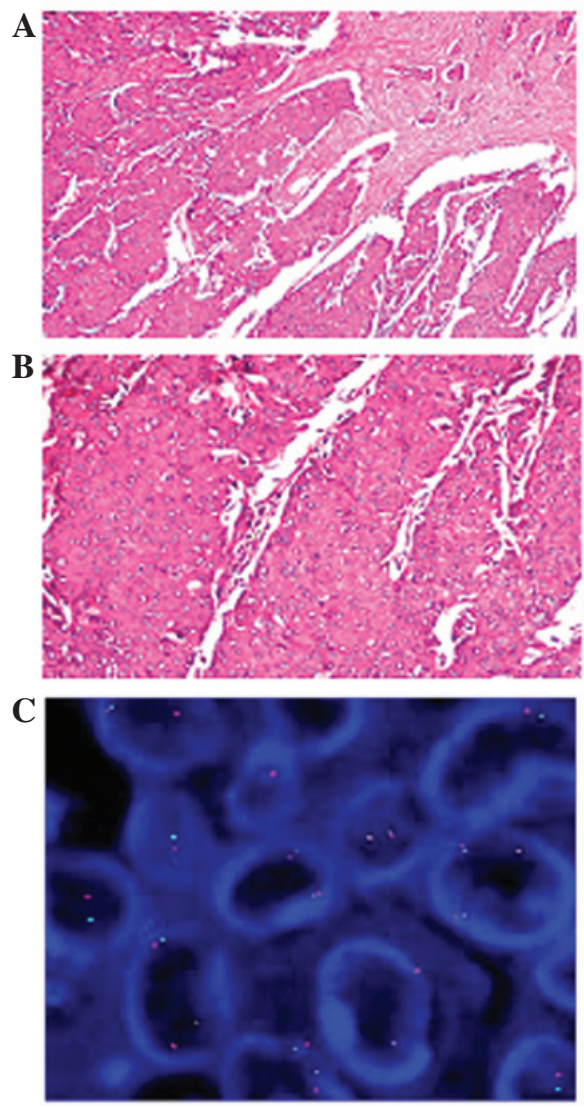

Figure 7. Right breast biopsy. (A) Hematoxylin and eosin staining; original magnification, $x 40$. (B) Hematoxylin and eosin staining; original magnification, $x 400$. (C) Fluorescence in situ hybridization analysis showing the presence of anaplastic lymphoma receptor tyrosine kinase rearrangement; original magnification, $\mathrm{x} 1,000$.

\section{Discussion}

Bronchogenic carcinoma is the foremost cause of tumor-associated mortality in developed countries (1). Women with
A

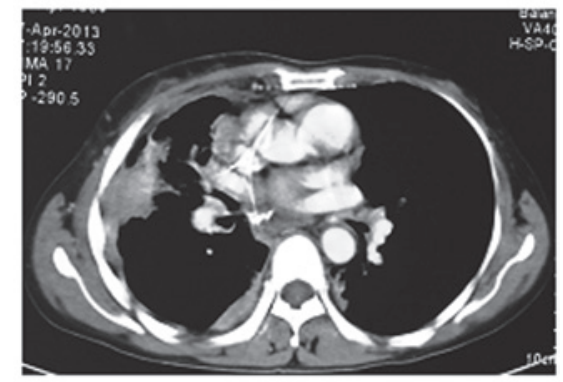

B

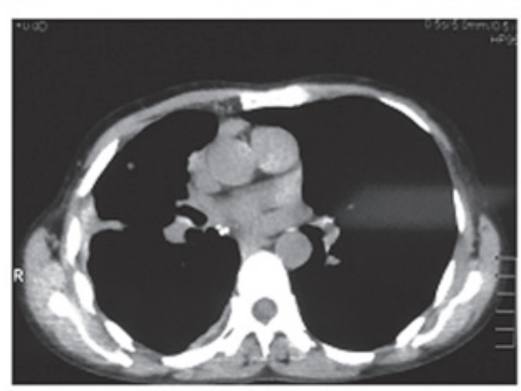

Figure 8. Partial remission (PR) acheived following crizotinib treatment. (A) Computed tomography scan of the chest after multiple lines of treatment. (B) PR after crizotinib treatment

metastatic ovarian adenocarcinoma from the lung have been reported to have a mean age of 46 years (3), with disease onset at a young age also being a prominent characteristic of $A L K$ rearrangement-positive NSCLC (4). The ovaries are an uncommon location for metastasis from lung cancer, and few such cases have been reported. The patients in the present study developed ovarian metastasis at different stages during their illnesses, and 1 patient developed breast metastasis. Ovarian metastasis from lung cancer represents only 2-4\% of all ovarian metastatic masses; however, this rate is increasing due to the rising occurrence of lung cancer in women (3). Secondary metastatic ovarian tumor occurrence is variable and depends on a number of factors, including the accuracy of the pathological diagnosis, the completeness of staging and possible 
genetic patterns. In the study by Young and Scully (5), 7 cases of ovarian metastasis of lung cancer. The study consisted of cases of ovarian tumors that were detected prior to $(n=3)$, concurrent with $(n=3)$ or $<1$ year after $(n=1)$ the primary lung cancer diagnosis. It was also indicated that the pathological features and clinical characteristics of the disease may be of use in the diagnosis of ovarian metastasis from lung cancer.

The occurrence rate of ovarian metastases derived from non-gynecological sites is 11 times greater than that of metastases from the female genital tract organs, with adenocarcinomas of the gastrointestinal tract being the most common (6). Kim et al discussed the cases of 166 patients with non-gynecologic malignancies and adnexal tumors, in which ovarian metastatic tumors were detected in $68 \%$ (7). Another large study reported that only $10 \%$ of 10,288 malignant ovarian neoplasms were metastatic (8).

Adenocarcinoma accounts for $\sim 33 \%$ of the lung carcinomas that metastasize to the ovary. Metastatic lung adenocarcinoma mimic ovarian surface epithelial-stromal tumors (particularly those large ovarian masses mimicking primary tumors), such as serous, endometrioid, mucinous and clear cell type tumors (3). Therefore, staining for CK-7 and CK-20 has widely been used to differentiate between primary and secondary ovarian malignancies. Yeh et al were the first to point out the importance of immunohistochemical staining (10). Positive TTF-1 and CK-7 staining, and negative CK-20 staining is diagnostically required to determine the correct diagnosis. The lungs and thyroid gland express TTF-1, a member of the NKx2 homeodomain transcription factor family, and this knowledge is widely used in surgical pathology, as well as in the determination of whether an adenocarcinoma of unknown primary originates from the pulmonary system (11). Napsin A may be a potential addition to the immunohistochemical panel for identifying lung cancer metastases. Napsin A is an aspartic proteinase detected in alveolar macrophages and type 2 pneumocytes, and a putative marker for pulmonary adenocarcinomas. Therefore, it may be of use in differentiating between primary lung adenocarcinoma and adenocarcinomas of other organs at the primary and metastatic sites (12). In particular, the combined use of napsin A and TTF-1 may be of great assistance due to the resultant increased sensitivity and specificity for identifying the lung origin of a metastatic adenocarcinoma (13).

In NSCLC patients with activating EGFR mutations, EGFR-specific tyrosine kinase inhibitors (TKI) are known to be an effective mode of therapy that is well tolerated. However, progression or relapse is inevitable during treatment in EGFR-TKI patients with EGFR-mutated NSCLC due to the appearance of drug resistance. This was evident in case 1 in the present study, where the patient developed progressive disease in the contralateral ovary following erlotinib treatment for 16 months. In half of all cases, a T790M point mutation is the cause of acquired resistance, which is believed to increase the affinity of EGFR for adenosine triphosphate (14).

Patients with $A L K$ rearrangements tend to be younger than the majority of patients with NSCLC (15). Echinoderm microtubule-associated protein-like 4 (EML4)-ALK rearrangements also occur more often in adenocarcinomas of individuals who have never smoked or those who are light smokers with tumors lacking EGFR and KRAS proto-oncogene, GTPase (KRAS) mutations. The incidence of $A L K$ rearrangement is only $3-5 \%$ in randomly selected NSCLC patients (16). EML4-ALK is a fusion-type protein tyrosine kinase found in 4-5\% of NSCLC cases $(4,17,18)$. The $A L K$ gene arrangements are largely mutually exclusive with EGFR or KRAS mutations (19). Screening for this fusion gene in NSCLC is important, as $A L K$-positive tumors are highly sensitive to therapy with $A L K$-targeted inhibitors. Crizotinib is the first Food and Drug Administration (FDA)-approved ALK TKI. The drug has been sanctioned for the treatment of locally advanced or metastatic NSCLC in those individuals with ALK-positive tumors, as determined using an FDA-approved test (20). Patients who present with advanced NSCLC that is positive for $A L K$ have been shown to exhibit objective response rates of $50-61 \%$ in single-arm clinical studies $(20,21)$. One case of bilateral ovarian metastasis of NSCLC with $A L K$ rearrangement has previously been reported, in which the patient benefited from crizotinib therapy, as determined by a lack of progression on regular follow-up examinations for 4 years after the initial surgery for the primary lung cancer (22). The patient in case 2 of the present study also benefited from crizotinib following multiline treatment modalities. The purpose of the present study, therefore, is to present two rare cases of ovarian metastasis with EGFR mutation and $A L K$ rearrangement-positive NSCLC, and to show how individual treatment can prolong the progression-free survival time.

Patients who have ovarian metastasis of NSCLC are rare, and those with EGFR mutation and $A L K$ rearrangement-positive ovarian metastasis are even rarer. The present study will aid our understanding of this type of metastasis, and will make physicians aware of the possibility of breast and ovarian metastasis in $A L K$-positive NSCLC patients.

\section{References}

1. Botana Rial M, Fernández-Villar A, González Piñeiro A and Leiro Fernandez V: Primary lung adenocarcinoma with ovarian metastasis: A rare presentation of bronchogenic carcinoma. Arch Bronconeumol 45: 571-572, 2009 (In Spanish).

2. Islami F, Torre LA and Jemal A: Global trends of lung cancer mortality and smoking prevalence. Transl Lung Cancer Res 4: 327-338, 2015.

3. Irving JA and RH Y: Lung carcinoma metastatic to the ovary: A clinicopathologic study of 32 cases emphasizing their morphologic spectrum and problems in differential diagnosis. Am J Surg Pathol 29: 997-1006, 2005.

4. Soda M, Choi YL, Enomoto M, Takada S, Yamashita Y, Ishikawa S, Fujiwara S, Watanabe H, Kurashina K, Hatanaka $\mathrm{H}$, et al: Identification of the transforming EML4-ALK fusion gene in non-small-cell lung cancer. Nature 448: 561-566, 2007.

5. Young RH and Scully RE: Ovarian metastases from cancer of the lung: Problems in interpretation - a report of seven cases. Gynecol Oncol 21: 337-350, 1985.

6. Vang R and Ronnett BM: Metastatic and miscellaneous primary tumors of the ovary. In: Gynecologic Pathology. Nucci MR and Oliva E (eds). Elsevier Churchill Livingstone, Philadelphia, pp539-614, 2009.

7. Kim K, Cho SY, Park SI, Kang HJ, Kim BJ, Kim MH, Choi SC, Ryu SY and Lee ED: Risk of metastatic ovarian involvement in nongynecologic malignancies. Int J Gynecol Cancer 22: 3-8, 2012 .

8. Shi Y, Ye D, Lu W, Zhao C, Xu J and Chen L: Histological classification in 10288 cases of ovarian malignant tumors in China. Zhonghua Fu Chan Ke Za Zhi 37: 97-100, 2002 (In Chinese).

9. American Joint Committee on Cancer: Lung Cancer Staging. 7th edition. http://cancerstaging.org/references-tools/quickreferences/documents/lungmedium.pdf. Accessed March 7, 2015. 
10. Yeh KY, Chang JW, Hsueh S, Chang TC and Lin MC: Ovarian metastasis originating from bronchioloalveolar carcinoma: A rare presentation of lung cancer. Jpn J Clin Oncol 33: 404-407, 2003.

11. Ordóñez NG: Thyroid transcription factor-1 is a marker of lung and thyroid carcinomas. Adv Anat Pathol 7: 123-127, 2000.

12. Suzuki A, Shijubo N, Yamada G, Ichimiya S, Satoh M, Abe S and Sato N: Napsin A is useful to distinguish primary lung adenocarcinoma from adenocarcinomas of other organs. Pathol Res Pract 201: 579-586, 2005.

13. Bishop JA, Sharma R and Illei PB: Napsin A and thyroid transcription factor-1 expression in carcinomas of the lung, breast pancreas, colon, kidney, thyroid, and malignant mesothelioma. Hum Pathol 41: 20-25, 2010.

14. Soria JC, Mok TS, Cappuzzo F and Jänne PA: EGFR-mutated oncogene-addicted non-small cell lung cancer: Current trends and future prospects. Cancer Treat Rev 38: 416-430, 2012.

15. Shaw AT, Yeap BY, Mino-Kenudson M, Digumarthy SR, Costa DB Heist RS, Solomon B, Stubbs H, Admane S, McDermott U, et al: Clinical features and outcome of patients with non-small-cell lung cancer who harbor EML4-ALK. J Clin Oncol 27: 4247-4253, 2009.

16. Solomon B, Varella-Garcia M and Camidge DR: ALK gene rearrangements: A new therapeutic target in a molecularly defined subset of non-small cell lung cancer. J Thorac Oncol 4: 1450-1454, 2009.
17. Mano H: Non-solid oncogenes in solid tumors: EML4-ALK fusion genes in lung cancer. Cancer Sci 99: 2349-2355, 2008.

18. Horn L and Pao W: EML4-ALK: Honing in on a new target in non-small-cell lung cancer. J Clin Oncol 27: 4232-4235, 2009.

19. Takahashi T, Sonobe M, Kobayashi M, Yoshizawa A, Menju T, Nakayama E, Mino N, Iwakiri S, Sato K, Miyahara R, et al: Clinicopathologic features of non-small-cell lung cancer with EML4-ALK fusion gene. Ann Surg Oncol 17: 889-897, 2010.

20. XALKORI ${ }^{\circledR}$ : (crizotinib) (package insert). Pfizer, NY, 2012. http://www.accessdata.fda.gov/drugsatfda docs/label/2012/202570s002lbl.pdf.

21. Kwak EL, Bang YJ, Camidge DR, Shaw AT, Solomon B, Maki RG, Ou SH, Dezube BJ, Jänne PA, Costa DB, et al: Anaplastic lymphoma kinase inhibition in non-small-cell lung cancer. N Engl J Med 363: 1693-1703, 2010.

22. Fujiwara A, Higashiyama M, Kanou T, Tokunaga T, Okami J, Kodama K, Nishino K, Tomita Y and Okamoto I: Bilateral ovarian metastasis of non-small cell lung cancer with ALK rearrangement. Lung Cancer 83: 302-304, 2014. 